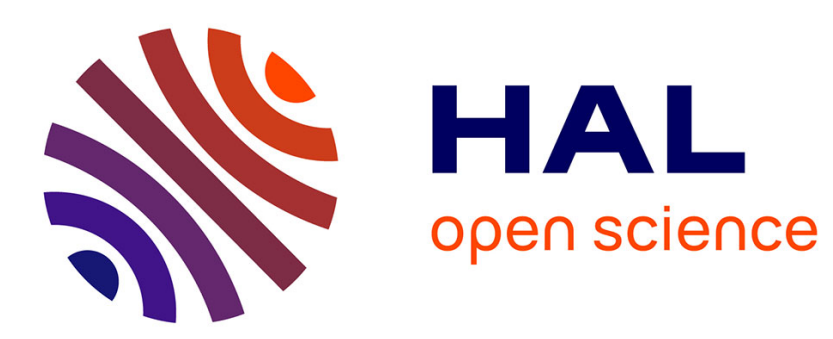

\title{
Spectroscopy of neutral and charged exciton states in single CdSe/ZnS nanocrystals
}

\author{
Yann Louyer, Louis Biadala, Philippe Tamarat, Brahim Lounis
}

\section{To cite this version:}

Yann Louyer, Louis Biadala, Philippe Tamarat, Brahim Lounis. Spectroscopy of neutral and charged exciton states in single CdSe/ZnS nanocrystals. Applied Physics Letters, 2010, 96, pp.203111. 10.1063/1.3435464. hal-00611568

\section{HAL Id: hal-00611568 \\ https://hal.science/hal-00611568}

Submitted on 26 Jul 2011

HAL is a multi-disciplinary open access archive for the deposit and dissemination of scientific research documents, whether they are published or not. The documents may come from teaching and research institutions in France or abroad, or from public or private research centers.
L'archive ouverte pluridisciplinaire HAL, est destinée au dépôt et à la diffusion de documents scientifiques de niveau recherche, publiés ou non, émanant des établissements d'enseignement et de recherche français ou étrangers, des laboratoires publics ou privés. 


\title{
Spectroscopy of Neutral and Charged Exciton States in Single CdSe/ZnS Nanocrystals
}

\author{
Yann Louyer, Louis Biadala, Philippe Tamarat, and Brahim Lounis *
}

Centre de Physique Moléculaire Optique et Hertzienne, Université de Bordeaux and CNRS, 351 cours de la Libération, Talence, F-33405 (France)

*blounis@u-bordeaux1.fr

\begin{abstract}
We report a low temperature spectroscopic study of the lowest-energy exciton states in single $\mathrm{CdSe} / \mathrm{ZnS}$ colloidal nanocrystals. In their neutral charge state, the zero-phonon lines of the lowest two levels of the band-edge exciton fine structure are directly observed in the photoluminescence spectrum. The attribution of these lines is confirmed by polarization-resolved studies of the photoluminescence spectrum and decay. We also identified the emission from charged nanocrystals which displays a single zero-phonon line $\sim 17 \mathrm{meV}$ red-shifted with respect to the neutral nanocrystal emission lines. This bright trion state has a lifetime which ranges from 1.5 to $4.5 \mathrm{~ns}$.
\end{abstract}


Quantum-confined nanoparticles have been increasingly investigated over the past decade due to the superior efficiency and tunability of their emission wavelength from the ultraviolet to the near infra red. Among those nanoparticles, colloidal CdSe nanocrystals (NC) are particularly attractive for many applications such as nanoscale electronics [1-3], laser technology [4], and quantum cryptography [5, 6]. These applications require a detailed understanding of both the spectral and polarization properties of $\mathrm{NC}$ emission and, specifically, the structure of the lowest-energy band-edge exciton states.

In neutral and spherical colloidal CdSe NCs, the band-edge exciton is split by the intrinsic electron-hole exchange interaction and by the crystal field of the hexagonal lattice into five energy levels [7]. The exciton ground state $|F\rangle$, which is often referred to as the "dark" exciton ( $J= \pm 2)$, is located a few meV below the lowest-energy optically active exciton state $|A\rangle$. Recent investigations of low temperature PL spectra from individual CdSe NCs led to the observation of a zero-phonon-line (ZPL) doublet with an energy splitting in the meV range [8-10]. Different attributions of these sharp emission lines were proposed. Htoon at al. [8] found that the two lines are characterized by linearly and orthogonally polarized emission dipoles, and attributed them to emission from two linear combinations of the $\mathrm{J}= \pm 1^{\mathrm{L}}$ bright exciton sublevels. They suggested that the splitting originates from an anisotropic electron-hole exchange interaction resulting from a breakdown of cylindrical symmetry in the NCs. This distortion-induced splitting is often referred to as the $\mathrm{X}-\mathrm{Y}$ splitting and has been well known for epitaxial quantum dots [11-14]. On the other hand, the ZPL doublet reported in Refs $[9,10]$ has been attributed to the radiative recombination from the lowest bright and dark states of the band-edge exciton fine structure. In this letter, we report single CdSe/ZnS NCs polarization- and time-resolved PL studies which fully support the latter assignment. For some NCs, we also identified transitions to a charged exciton state (trion), and measured for the first time this trion lifetime on single NCs.

Samples of CdSe/ZnS core-shell NCs (Qdot655 from Quantum Dot Corp., peak emission at 655 $\mathrm{nm}$ at room temperature) are prepared by spin coating clean glass coverslips with a nanomolar solution of NCs in polyvinyl alcohol. A homebuilt scanning confocal microscope is used to image single NCs 
excited with the $532 \mathrm{~nm}$ line of a cw frequency doubled Nd:YAG laser. It is based on a 0.95 NA objective (diffraction-limited laser spot size of $\sim 0.4 \mu \mathrm{m}^{2}$ ) which is inserted in a helium cryostat together with the sample and a piezoscanner. The emitted photons are filtered from the scattered excitation light by a bandpass filter (60 $\mathrm{nm}$ FWHM) and sent to a single-photon-counting avalanche photodiode and a spectrometer. The PL decay measurements are performed with a conventional time correlated single photon counting setup, using a pulsed laser source (optical parametric oscillator at $570 \mathrm{~nm}, 6 \mathrm{ps}$ pulse width, $76 \mathrm{MHz}$ repetition rate) and a pulse picker which reduces the repetition rate.

At $2 \mathrm{~K}$, most of the single NCs are photostable and do not show any blinking. Figure 1 (a) shows an example of individual NC spectra displaying two sharp resolution-limited ZPLs. At $2 \mathrm{~K}$, most of the luminescence $(\sim 90 \%)$ stems from the low energy ZPL (denoted F) with its longitudinal acoustic (LA) and optic (LO) phonon replica. This ZPL is attributed to the dark exciton radiative recombination. When raising the temperature from $2 \mathrm{~K}$ to $10 \mathrm{~K}$, the high energy ZPL (A) largely prevails over the $\mathrm{F}$ line. This striking observation supports a phonon-mediated thermal mixing between two emitting levels which have very different oscillator strengths, and therefore different spin natures (namely $\mathrm{J}=1$ and $\mathrm{J}=2$ ). Furthermore, one can clearly notice at $3.5 \mathrm{~K}$ (Fig. 1 (b)) that the relative weights of the LO-phonon sidebands with respect to the ZPLs are significantly different for the two states. This supports the assumption that radiative recombination from the dark state occurs preferentially with emission of phonons.

Figure 1 (e) shows the PL decay of this single NC recorded at $2 \mathrm{~K}$. The decay shows a biexponential behavior with a fast decay occurring within a ns and a much slower one with a lifetime of hundreds of ns. The long time component of the decay drastically shortens with temperature, from $\sim 700$ $\mathrm{ns}$ at $2 \mathrm{~K}$ to $10 \mathrm{~ns}$ at $92 \mathrm{~K}$ (inset of Fig. 1(e)). This temperature dependence shows evidence for a thermal mixing between two excitonic states having a two orders of magnitude difference in their decay rates [15]. It can be reproduced within the frame of a three-level model taking into account the ground state and the two thermally mixed emitting states [9]. The fit (solid curve) of the experimental data gives the bright state radiative decay rate $0.22 \mathrm{~ns}^{-1}$, and the bright-to-dark spin flip rate $1.0 \mathrm{~ns}^{-1}$. This reduced 
spin relaxation rate is due the small phonon density of the states at the scale of the inter-level splitting [9]. The weight of the long component represents $\sim 90 \%$ of the PL decay at $2 \mathrm{~K}$. One can deduce that it arises from the dark exciton radiative recombination since the $\mathrm{F}$ line and its phonon replica correspond to the same fraction $(\sim 90 \%)$ in the luminescence spectrum. Such observations cannot be reproduced using a model with two X,Y bright states having oscillator strengths of the same order of magnitude.

We also performed polarization-resolved studies of the PL spectra and the PL decay for several single NCs, using a half-wave plate and a Glan polarizer on the PL beam pathway. As examplified in Fig. 2, we observed various behaviors which probably result from different relative orientations between the NC hexagonal axis and the laser field. For ten NCs studied, we could not find a single NC for which the A and F lines are linearly and orthogonally polarized, as one would expect if these lines stemmed from the X,Y states. Furthermore, we could not attenuate the intensity of the F line by more than a factor of 2 upon rotating the half wave plate, whereas the intensity of the A line could be modified by a larger factor (e.g. $\sim 4$ for the $\mathrm{NC}$ of Fig. 2 (a), 10 for the one of Fig. 2 (b)). According to theoretical calculations using the pseudopotential configuration-interaction method [16], the emission from the bright exciton is polarized perpendicular to the hexagonal $\mathrm{NC}$ axis, whereas the degree of linear polarization of the dark exciton decreases with the $\mathrm{NC}$ size as a result of an increasing polarization component along the crystal axis. Our polarization measurements therefore support the attribution of the F line to the long-lived dark exciton state. Moreover, polarized decay measurements show that the weight of the short time component follows that of the A line of the polarized emission spectrum.

Another important result obtained with these NCs is the spectroscopic signature of a charged excitonic state. Among hundreds of single NCs studied, we found $17 \mathrm{NCs}$ for which the emission switched between the A, F doublet and a red-shifted single ZPL X* (Fig. 3 (a)). We attribute such spectral jumps to transitions between neutral and charged excitonic states. A trion state is indeed prepared when an exciton is created in the presence of an unpaired charge carrier in the NC core and a charge carrier trapped at the surface or in a lattice defect of the NC. As displayed in the inset of Fig. 3 (a), the distribution of spectral shifts between the dark exciton ZPL and the trion ZPL ranges from 15.5 
to $18.5 \mathrm{meV}$. Similar spectral jumps have been previously reported for single CdSe nanorods with a $\sim 2.5$ $\mathrm{nm}$ radius and $\mathrm{a} \sim 25 \mathrm{~nm}$ length [17], as well as for self assembled CdSe quantum dots [18]. Yet, much smaller jumps (a few meV) have been recently observed by Fernée et al. on single NCs with a $\sim 4 \mathrm{~nm}$ radius [10]. The bright trion ZPL is accompanied by a weak LO-phonon replica (weight lower than 7\%, data not shown) and confined acoustic phonon sidebands. As shown in Fig. 3 (a), one can distinguish two LA-phonon replica, $1.1 \mathrm{meV}$ and $1.8 \mathrm{meV}$ redshifted from the $\mathrm{X}^{*} \mathrm{ZPL}$, which we attribute to acoustic radial breathing modes of the NC [19-21].

We also recorded the PL decay of single NCs which were permanently charged. As shown in Fig. 3 (b), the decay is monoexponential with a relaxation time in the range 1.5-4.5 ns with mean value 3.0 ns. These decay times are shorter than the radiative lifetime of the neutral bright exciton (lying in the range 4.5-7.4 ns with a mean value of $6.2 \mathrm{~ns}$, data not shown). This suggests that Auger recombination processes contribute to the charged exciton decay rate. According to pseudo-potential configurationinteraction calculations [16], a single red-shifted ZPL associated to a short lifetime emission (in the nanoseconds range) is a signature of the negative trion state. Recent ensemble measurements on CdSe NCs with a comparable size provided a value of $\sim 1 \mathrm{~ns}$ for the negative trion lifetime, using an indirect deconvolution method to extract the trion lifetime from multiexponential decays [22].

In summary, our spectroscopic investigations of single CdSe/ZnS NCs demonstrated that the ZPL doublet observed in the low temperature PL spectra correspond to the radiative recombination from the two lowest levels of the band edge exciton fine structure. We also determined the characteristic features of a bright charged state attributed to the negative trion state. Further investigation will aim at determining the optical coherence lifetime of the trion ZPL emission for applications in quantum optics, such as the realization of indistinguishable single photon sources. 
Acknowledgement. This research was funded by the Région Aquitaine, the Agence Nationale pour la Recherche (ANR), the French Ministry for Education and Research (MESR) and the European Research Council (ERC). 
References.

[1] V. L. Colvin, M. C. Schlamp, and A. P. Alivisatos, Nature 370, 354 (1994).

[2] B. O. Dabbousi et al., Applied Physics Letters 66, 1316 (1995).

[3] M. C. Schlamp, X. G. Peng, and A. P. Alivisatos, Journal of Applied Physics 82, 5837 (1997).

[4] V. I. Klimov et al., Science 290, 314 (2000).

[5] P. Michler et al., Science 290, 2282 (2000).

[6] B. Lounis et al., Chemical Physics Letters 329, 399 (2000).

[7] A. L. Efros et al., Physical Review B 54, 4843 (1996).

[8] H. Htoon et al., Physical Review B 77, 035328 (2008).

[9] L. Biadala et al., Physical Review Letters 103, 037404 (2009).

[10] M. J. Fernée, B. N. Littleton, and H. Rubinsztein-Dunlop, Acs Nano 3, 3762 (2009).

[11] M. Bayer et al., Physical Review Letters 82, 1748 (1999).

[12] D. Gammon et al., Physical Review Letters 76, 3005 (1996).

[13] V. D. Kulakovskii et al., Physical Review Letters 82, 1780 (1999).

[14] J. Puls et al., Physical Review B 60, R16303 (1999).

[15] O. Labeau, P. Tamarat, and B. Lounis, Physical Review Letters 90, 257404 (2003).

[16] M. Califano, A. Franceschetti, and A. Zunger, Physical Review B 75, 115401 (2007).

[17] N. Le Thomas et al., Phys Rev Lett 94, 016803 (2005).

[18] B. Patton, W. Langbein, and U. Woggon, Physical Review B 68, 125316 (2003).

[19] U. Woggon et al., Physical Review B 54, 1506 (1996).

[20] G. Chilla et al., Physical Review Letters 100, 057403 (2008).

[21] M. J. Fernée et al., Journal of Physical Chemistry C 112, 1878 (2008).

[22] P. P. Jha, and P. Guyot-Sionnest, Acs Nano 3, 1011 (2009). 

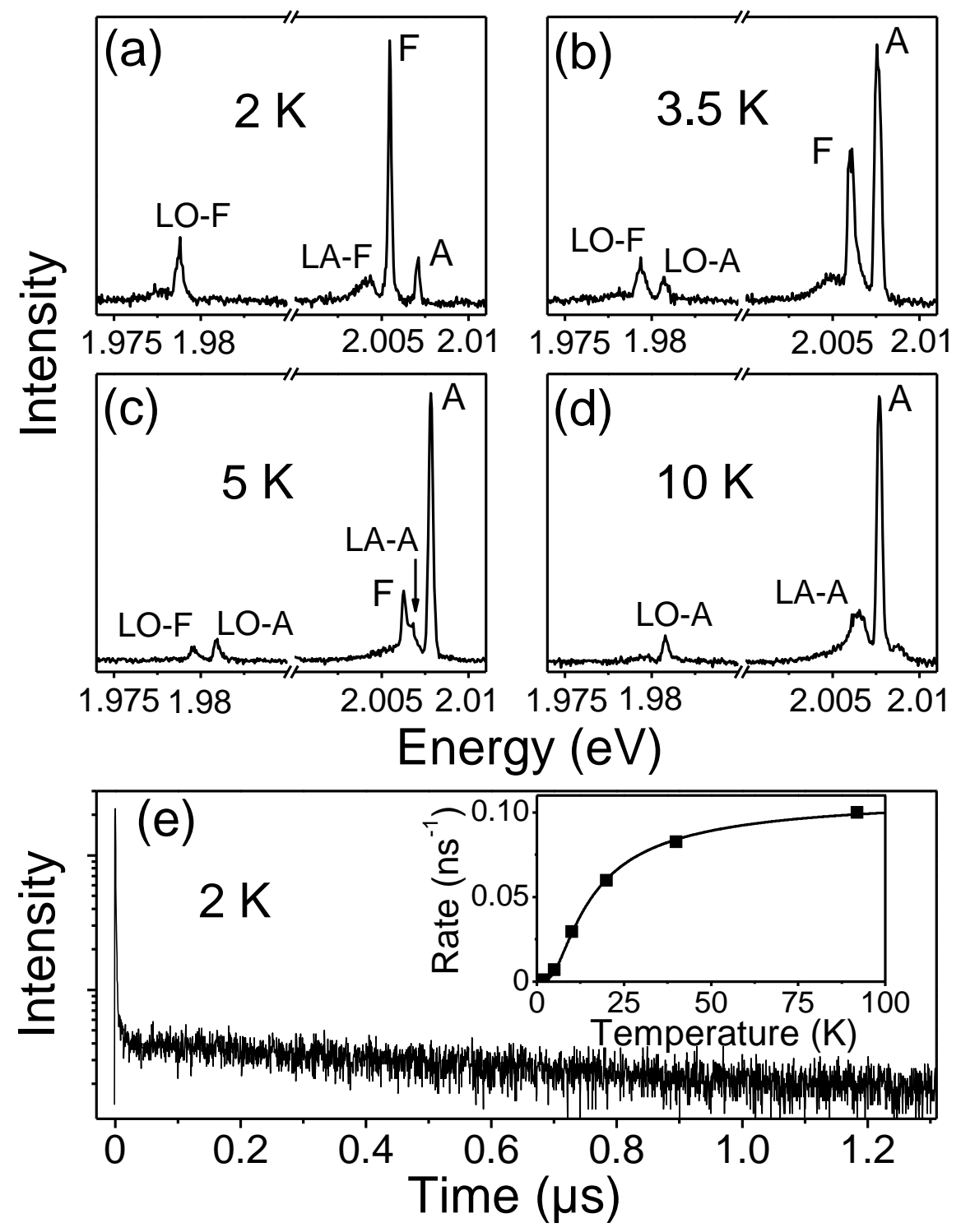

FIG 1. (a)-(d) Non polarized PL spectrum of a single CdSe/ZnS NC recorded at various temperatures, from $2 \mathrm{~K}$ to $10 \mathrm{~K}$, at a $\mathrm{CW}$ excitation intensity of $50 \mathrm{Wcm}^{-2}$. The peaks labelled $\mathrm{F}$ and $\mathrm{A}$ are attributed to the ZPLs of the dark and bright excitons, respectively. The peaks LO-F and LO-A are LO-phonon replica of the peaks F and A. The structures LA-F and LA-A are acoustic phonon replica of the peaks F and A. (e) Biexponential PL decay of the same $\mathrm{NC}$ at $2 \mathrm{~K}$ (peak excitation intensity $1 \mathrm{MW} \mathrm{cm}^{-2}$ ). The decay time of the long component is $~ 700 \mathrm{~ns}$. The evolution of the long component decay rate with temperature is shown in the inset (square dots). The solid curve is a fit using the model of reference [9]. 

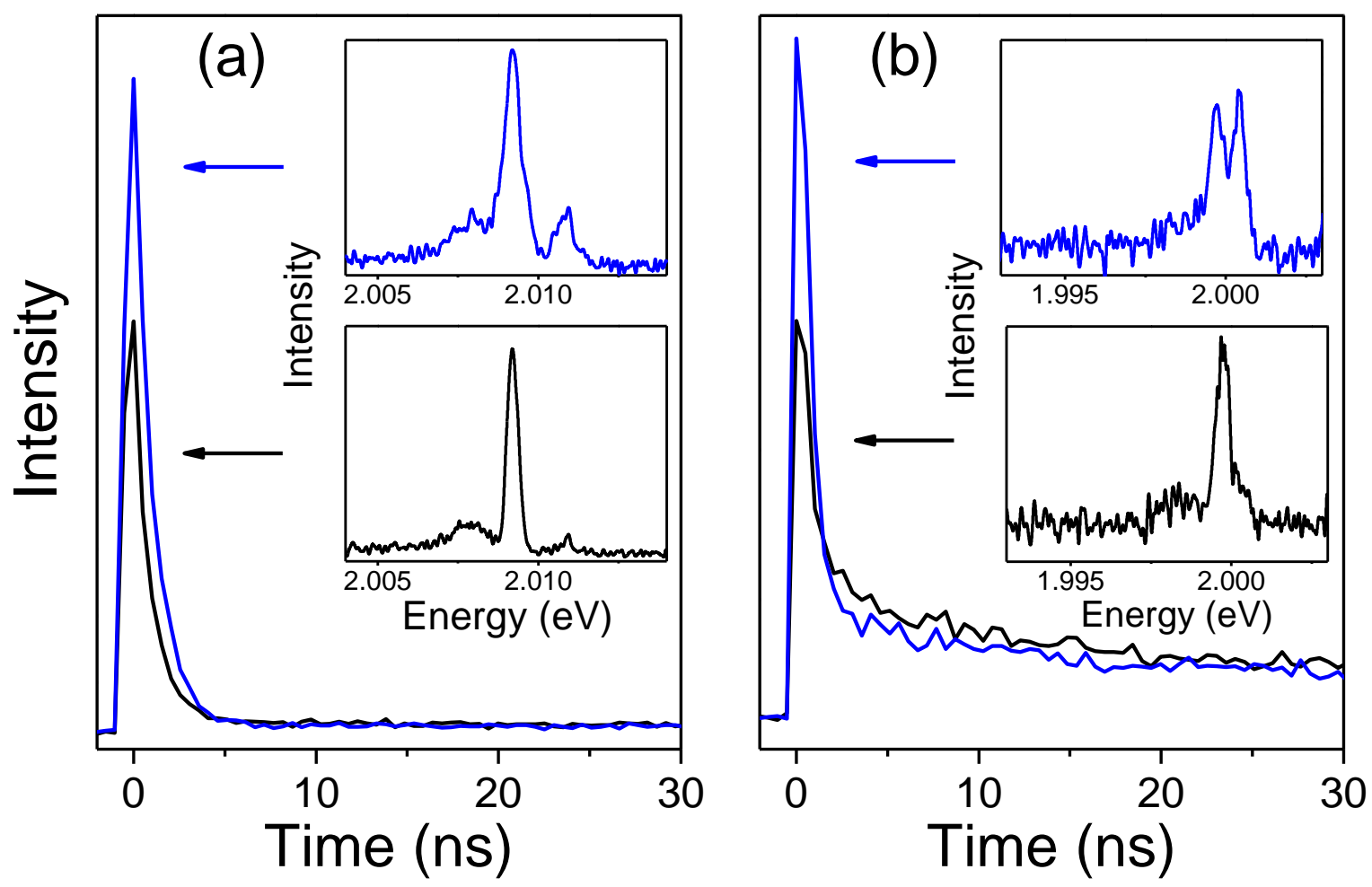

FIG. 2. Polarization-resolved emission spectrum and PL decay for two single NCs at $2 \mathrm{~K}$. The intensity scale is linear. (a) PL decay of the same single NC as in Fig. 1, for two different polarization components of the luminescence. The total number of counts is the same for both decay histograms $\left(1.3 \times 10^{5}\right.$ counts). The inset shows the corresponding emission spectra. (b) Same experiment for another single NC. 

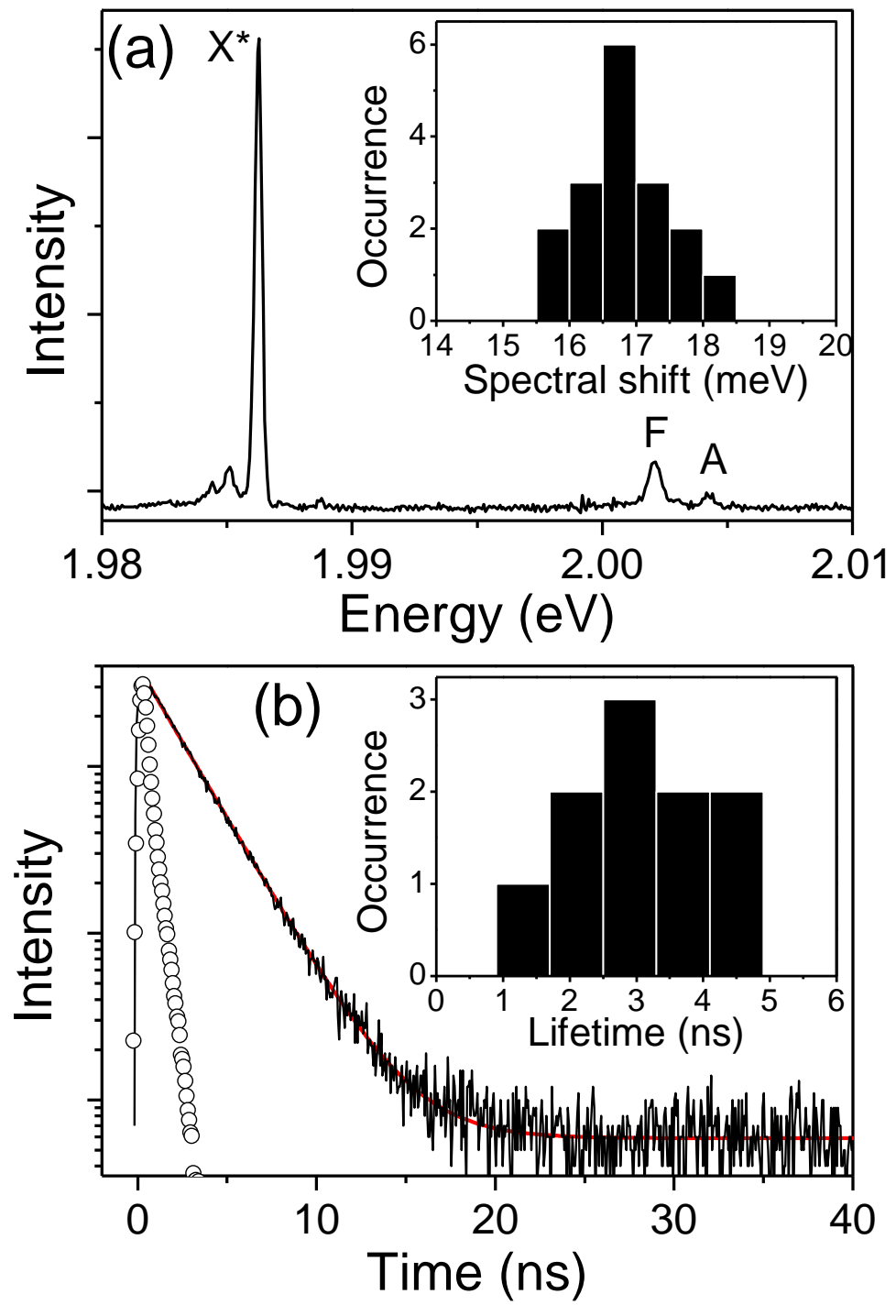

FIG. 3. (a) PL spectrum of a single $\mathrm{NC}$ at $2 \mathrm{~K}$, recorded over $20 \mathrm{~s}$ at an excitation intensity of $50 \mathrm{Wcm}^{-2}$. The peak labelled $\mathrm{X}^{*}$ is attributed to the ZPL of the lowest-energy charged exciton state. Inset: Histogram of the spectral shift between peaks $\mathrm{F}$ and $\mathrm{X}^{*}$, for 17 single NCs. (b) PL decay of a permanently charged single NC, at $2 \mathrm{~K}$. The solid curve fits the decay with a monoexponential law (decay time $2.4 \mathrm{~ns}$ ). The open circles correspond to the instrument response function. Inset: histogram of the trion lifetime, for 10 single NCs. 